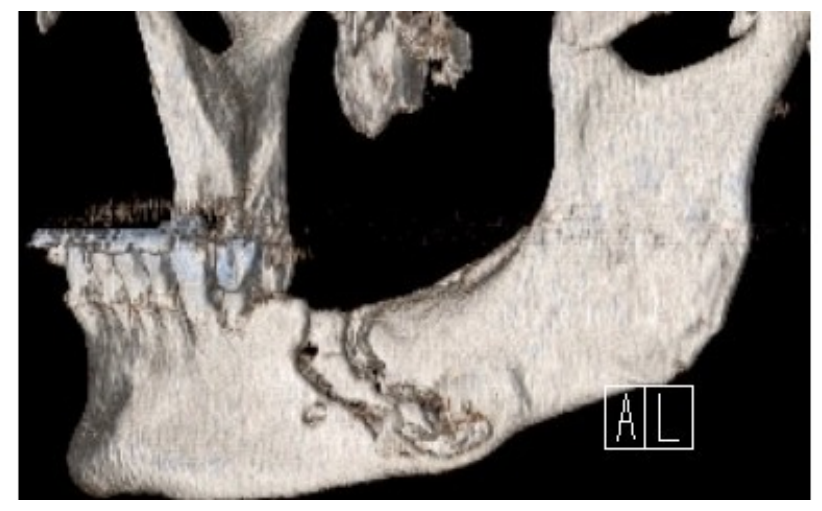

\title{
SURGICAL TREATMENT OF MEDICATION-RELATED OSTEONECROSIS OF THE JAW: TECHNICAL REPORT AND PROGNOSIS ASSESSMENT
}

\author{
Stefano Negrini ${ }^{1}$, Francesco Daleffe ${ }^{1}$, Elisabetta Audino ${ }^{2}$, luca visconti, Stefano Salgarello ${ }^{2}$, Andrea Castellani \\ 1 ASST Spedali Civili \\ 2 University of Brescia
}

Funding: The author(s) received no specific funding for this work.

Potential competing interests: The author(s) declared that no potential competing interests exist.

\section{Abstract}

Since the first reports concerning osteonecrosis of the jaws there has been a lot of debate about the treatment modalities: therapy of the established pathology can in fact be medical or surgical and, in the latter case, be conservative or radical. The widespread difference of clinical presentations and relative rarity of advanced stages disease at diagnosis has not yet been able to guarantee a complete standardization in the therapeutic planning phase. Although there is a well-defined staging of the pathology, the international debate is still heated about more or less invasive surgical attitudes.

We collected data about ONJ in oncological patients surgically treated in our centre during the last 14 years: particular attention was paid to the variables and correlations between relapse, disease staging and treatment modalities. This is to underline the correlation between stage and type of treatment, as well as the greater possibility of relapse in the more advanced stages, in addition to its constancy in relation to the types of treatment. Finally, particular attention was paid to the timing of the relapse, generally located in the second semester of follow-up.

Our clinical cases presentation, although far from statistical significance, aims to generate attention especially on this last parameter, in order to improve outpatient follow-up procedures. 


\section{Background}

Medication-Related OsteoNecrosis of the Jaws (MRONJ) is a bone pathology affecting those patients who, due to their specific systemic conditions and without previous actinic therapy, assume antiresorptive or anti-angiogenetics drugs. Although MRONJ is believed to be an avascular necrosis of the jaws, the precise specific aetiology remains unclear. The summentioned conditions lead to various clinical presentations, all characterized by superinfected bone necrosis: diagnosis must therefore be based on contemporary clinical and radiological data. ${ }^{[1][2]}$

Since the first reports concerning the disease, which are indeed relatively recent, there has been a lot of debate about the treatment modalities. The therapy of the established pathology can in fact be medical or surgical and, in the latter case, be conservative or radical. ${ }^{[3][4]}$ Recent years evidence has relegated medical therapy to increasingly early disease, demonstrating that only the surgical approach is decisive both in advanced and early cases, not responsive to medical therapy. In fact, the primary intent of surgery in drug-related ONJ should not be palliative, but curative. ${ }^{[1]}$ Also, accurate staging systems have been developed, with different descriptions of clinical and radiological features. Current SIPMO-SICMF classification defines three worsening stage: focal ONJ, limited to alveolar bone, diffuse ONJ, involving basal bone, and complicated ONJ, characterized by peculiar complications (such as pathological fracture, sinsual or cutaneous extension).

The widespread difference of clinical presentations and relative rarity of advanced stages disease at diagnosis has not yet been able to guarantee a complete standardization in the therapeutic planning phase. Although there is a well-defined staging of the pathology, the international debate is still heated about more or less invasive surgical attitudes:[5] this is even more true in those patients in whom bone segmental resection may appear excessive, while local sequestrectomy and debridement risk being insufficient. ${ }^{[6]}$ In fact, in a balance of surgery consequences and patient performance-status, it is not always easy to obtain the presence of histologically normal bone tissue (especially cortex from inferior mandibular border) at the resection margin.

Our clinical cases presentation, although far from statistical significance, aims to analyze the type of treatment our patients have undergone, their pathology stage and, above all, the relapse incidence and its timing in relation to the abovementioned parameters.

\section{Materials and methods}

Our database included patients with diagnosis of oncological pathology, in treatment with antiresorptive drugs, medically or surgically treated for jaw osteonecrosis in MaxilloFacial Department between 2006 january and 2020 july. We decided to exclude "metabolic" patients due to the difficulty in homogenizing the sample they represented.

All surgical procedures were executed by the same surgical team and according to the same surgical protocols: we performed a less invasive surgery ("A" treatment), by local sequestrectomy and debridement, or a more demanding approach ("B" protocol), with extensive marginal or segmental resection.

Indications for "A" treatment were mainly focal ONJ, with little cases of diffuse and complicated ONJ . Conditions requiring "B" operation were roughly the opposite; this attitude could appear partially in contrast with the sicmf-sipmo recommendations, but it concerns a sample extending in a time far preceding the same. Moreover we must often deal with patients' will, their general conditions and palliative necessity.

The patients were evaluated prospectively and investigated at least twice; the two examinations were at least 6 months 
apart, lasting till 24 months after surgery. Each patient underwent at least one first level control radiological investigation during the outpatient follow-up, generally through orthopantomography. In case of doubt a CT scan was prescribed; this to exclude relapses of initially asymptomatic pathology.

Exclusion criteria were the presence of confounding variables such as corticosteroids in medication, history of radiation, metastases within the gingiva or jaws.

Surgery was carried out 4 to 12 months after the last dose of antiresorptive or anti-angiogenetics drugs (mean 6,5 month), if those were not discontinued. Resumption of therapy was suggested no less than one month after surgery. This did not seem to cause any disturbances in wound healing. Histological examination of the bone and surrounding soft tissues was routinely performed to confirm the clinico-radiological diagnosis and to exclude metastatic disease.

Wound closure was carried out without tension on the local flap, interrupted sutures were performed with resorbable material (Vicryl 3-0/4-0, Ethicon, Norderstedt, Germany).

Once the data was collected, we proceeded with a descriptive analysis and, above all, with a comparison between the variables of the stage of disease, type of surgical intervention, relapse and timing of relapse. Recurrence was defined, according to SICMF-SIPMO positions, as presentation of signs or symptoms of illness in the same surgical site or in nearby locations within 12 months of primary treatment.

\section{Results}

Between 2006 january and july 2020 our Unit surgically treated 92 oncological patients with MRONJ due to their therapy, resulting from 346 patients evaluated on an outpatient basis. The number of total surgical procedures consisted in 105 treatments, due to $13 \mathrm{ONJ}$ recurrences. Postoperative follow-up lasted usually 2 years (time of discharge from outpatient checks) progressively shortening for the last patients treated in 2019 and 2020, up to a minimum of 10 months.

The age range of surgically treated patients was between 45 and 91 years (mean 63,16 years); our sample consisted of 27 male and 65 female, with a M/F ratio of 2.4/1. To be noted, more frequent primitive oncological pathology was breast cancer (40 cases), according to literature the most representative oncological disease in MRONJ (Tab 1). We also treated ONJ deriving from medical treatment of 36 patients with multiple myeloma, 9 prostate cancer and 7 of other origins. (Tab 2) The antiresorptive or anti angiogenic drug assumed was Zoledronate for 71 patients, while Denosumab was prescribed for 6 patients, Zoledronate followed by Denosumab in 6 patients, Pamidronate in 5 cases and Alendronate in 4 patients (Tab 3). Due to relatively recent widespread of monoclonal antibodies, and the possible remission of osteonecrosis following their suspension, only a small number of patients assuming Denosumab alone underwent surgery.

Based on current SICMF-SIPMO staging, 35 patients were classified as stage I (38,04\%), 30 as stage II ( $32,60 \%)$ and 27 patients as stage III (29,34\%) (Tab 4). In 23 patients (25.3\%), ONJ was located in the upper jaw, 67patients (73.6\%) were affected in the lower jaw, and 1 patient $(1,08 \%)$ developed $\mathrm{ONJ}$ in both jaws.

We classified our patients also for type of surgical treatment: 36 underwent debridement and / or sequestrectomy with bone remodeling (type "A" surgery) )while 56 were treated by marginal or full thickness bone resection (type "B" surgery). We have also divided our series in relation to both the stage of the disease and the type of treatment: the results are shown in table 5. More specifically, over 40 patients treated with "A" approach, 31 were classified as focal ONJ (stage I), while among the 52 patients undergoing type "B" surgery, 25 were stage II (diffuse ONJ) and 24 were type III (complicated ONJ). Finally we investigated the relapse rate, present in 13 of our patients, and its correlation with pathology stage: it consisted 
in 3 focal ONJ cases (12\% of the stage I sample), 4 diffuse ONJ patients (14.3\% of the stage II patients group) and 5 complicated ONJ (23.8\% of the stage III). Again, recurrence was related to type of surgery, with a quite stable ratio of $15,4 \%$ (4 cases) in type "A" treatment to 18\% (9 patients) in type "B" surgery. Concluding, while relying on limited data, we have tried to identify a tendency to relapse over time (episodes from 3 to 34 months postoperatively, with an average value of 11 months and a median of 7), confirmed residing in the second postoperative semester. (tab 6)

\section{Discussion and Conclusions}

According to literature, surgical therapy is effective in most patients suffering from MRONJ4; basing on aforementioned results, we can underline important prognostic factors related to pathology recurrence.

Analyzing all the results we can highlight how our sample is substantially aligned with the literature in numerous features: first of all, the female predominance and the mean age of presentation. ${ }^{[7]}$ For more than 10 years the evidence has agreed that MRONJ is a characteristic pathology of elderly age and of the female sex. This is due to the pathologies, metabolic as well as oncological, for which antiresorptive drugs are prescribed. From this point of view, the sample appears homogeneous also for primary pathology, mostly represented by breast cancer and multiple myeloma, followed at a distance by further neoplastic secondarisms. ${ }^{[8][9]}$ Being the oncological pathology aligned with the literature, and the treatment of the same highly standardized, it is evident that the antineoplastic treatment protocols are similar to what is reported in the scientific evidence: Zoledronate prescriptions therefore appear largely predominant . [10] Some other characteristics of our sample, however, are in contrast with what has been widely reported: first of all the relationship between outpatient assessments and surgical treatments (established around $26 \%$ ). This can easily derive from the valuable exertion of our Dental Clinic, active in an intense screening that therefore limits to only the most serious cases the influx to our attention.

Again, the large predominance of mandibular involvement appears to be minor in our cases, being the MRONJ present in $25.3 \%$ of cases in the maxilla, in $73.6 \%$ in the mandible and in $1 \%$ in both locations. In a substantially similar way, it can be appreciated an almost equal distribution of the sample studied in the three staging categories(Tipe I, II and III according to SICMF-SIPMO classification). ${ }^{[11][12]}$ According to our experience, the type of surgery must necessarily be tailored depending on the extent and location of the bone disease, the health of the soft tissues and, last but not least, the patient's performance status. These considerations lead to an asymmetrical division of the study group into the two types of surgery: early disease stages are generally treated with debridement or sequestrectomy (treatment-group "A"), advanced stages generally undergo bone resection (treatmentgroup “B”). 


\section{ONJ Staging compared to Type of treatmnet \\ stage I $\square$ stage II $\square$ stage III}

30

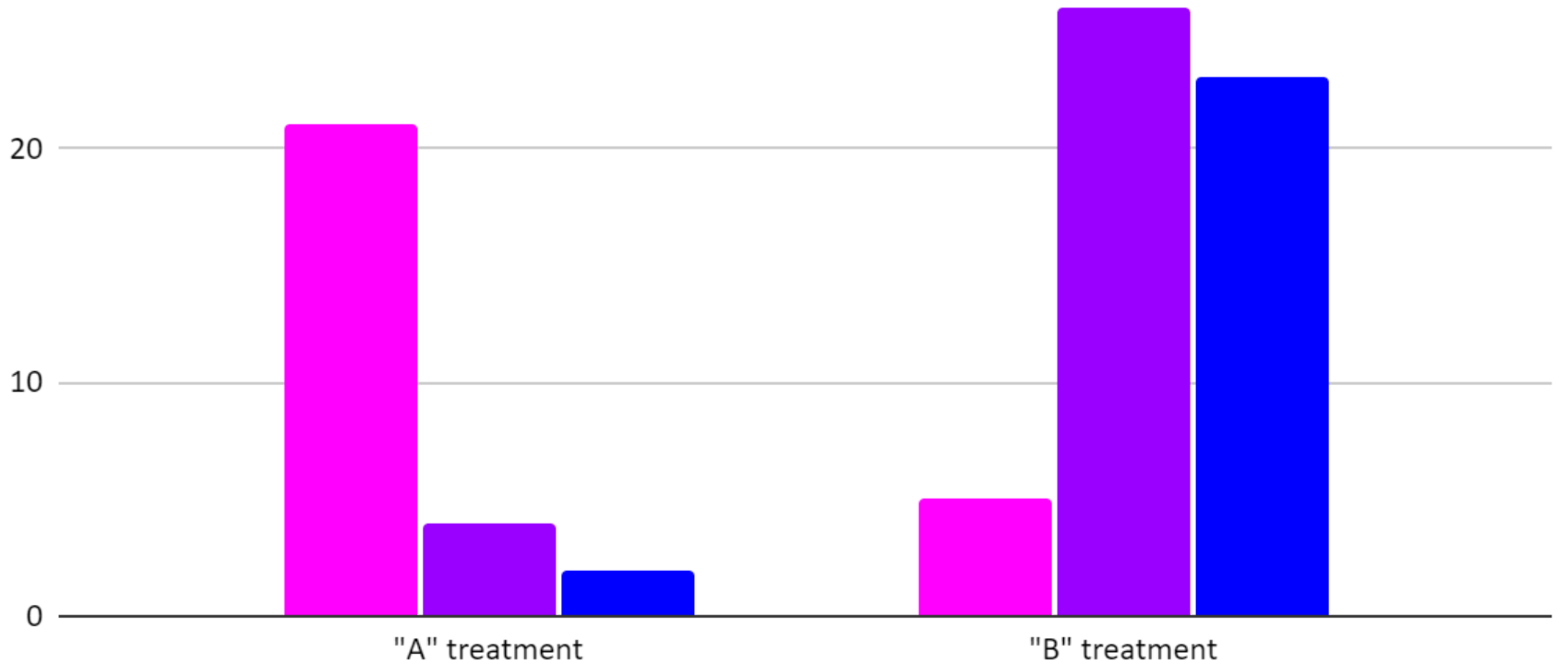

We must however consider that ONJ extensive resections have shown a high rate of success if the lesion was treated by segmental resections, above all in relapse. ${ }^{[13]}$ Although these results may be associated with a high success rate, patients are being subjected to quite radical treatment when often in a palliative phase of care; the undesirable effects of the resection (and possible reconstruction) should not be underestimated.

Our sample is characterized by a recurrence rate of $14,1 \%$, with 13 out of 92 cases re-operated. Literature highlights a quite wide range of values, varying from $22 \%$ to $18 \%$ to $9 \%{ }^{[14][15][16]}$ Tailoring the therapy and patient selection, in accordance with the most up-to-date clinical recommendations, still seem to be the most important factors to ensure the most favorable outcome 11.

In order to analyze the data more specifically, our intent is to correlate relapse and staging, recurrence and type of treatment and finally temporal trend of relapses. To our knowledge, although not statistically relevant, these associations have not often been extrapolated previously. ${ }^{[17]}$

The most evident data is the direct correlation between recurrence and staging of the disease: in fact as the former progressively increases, the tendency to relapse progressively worsens towards the third stage. 


\section{Relapse compared to ONJ stage}

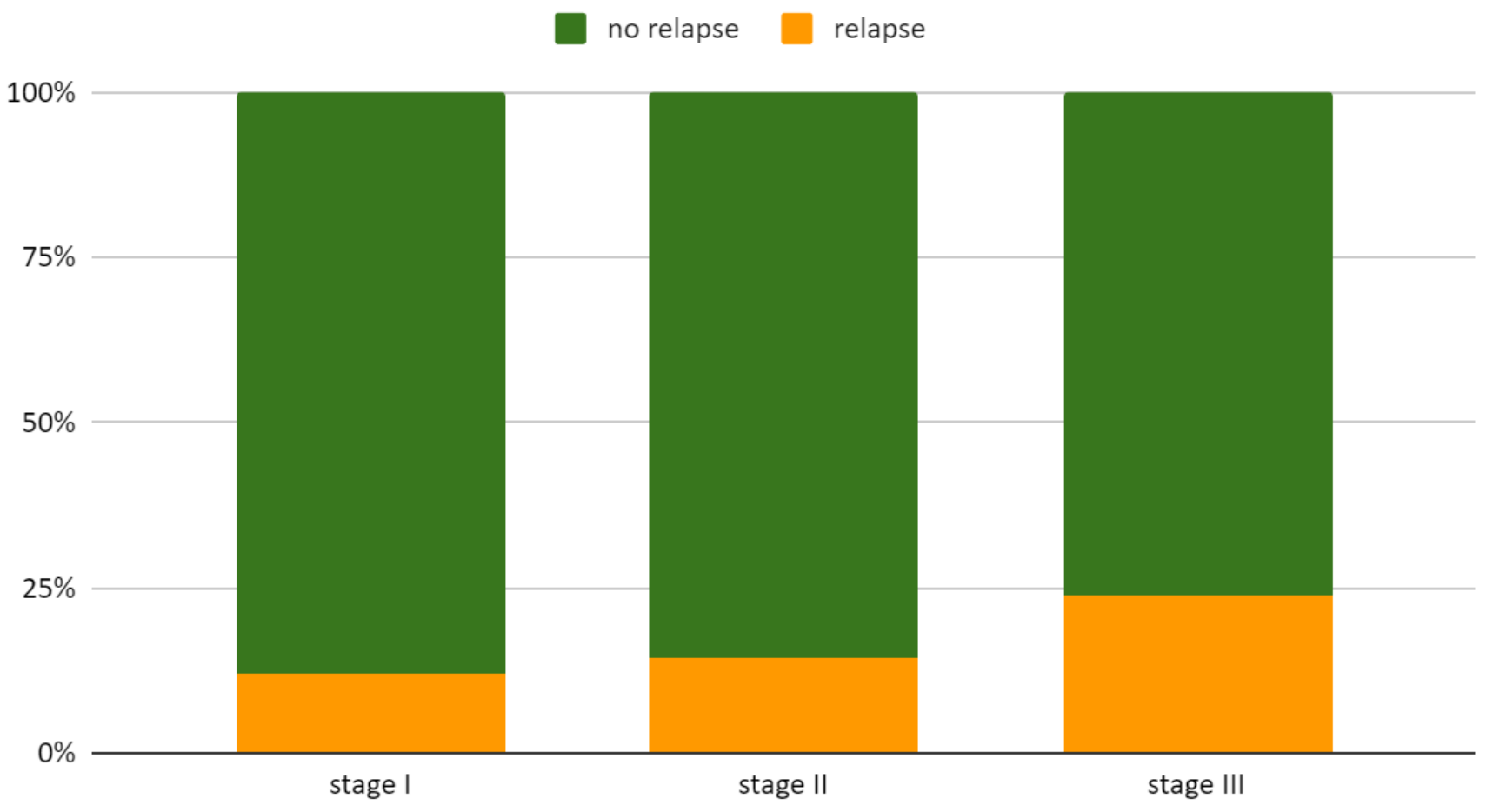

This could be explained by the fact that severe tissue alterations undergo extensive localizations of pathology, with severe prejudice of local soft tissues. This can easily hinder the repair process. Furthermore, despite careful pre-surgical planning, the operator is unconsciously led to a slightly more conservative attitude, especially during particularly extensive bone resections.

To the best of our knowledge, association between type of treatment and staging have not previously been clearly identified as prognostic factors for recurrence. Although many studies have been published since 2003 , controversy regarding the choice of surgical treatment is still heated. ${ }^{[1]}$ 


\section{Number of relapses compared to type of surgery \\ relapse no relapse}

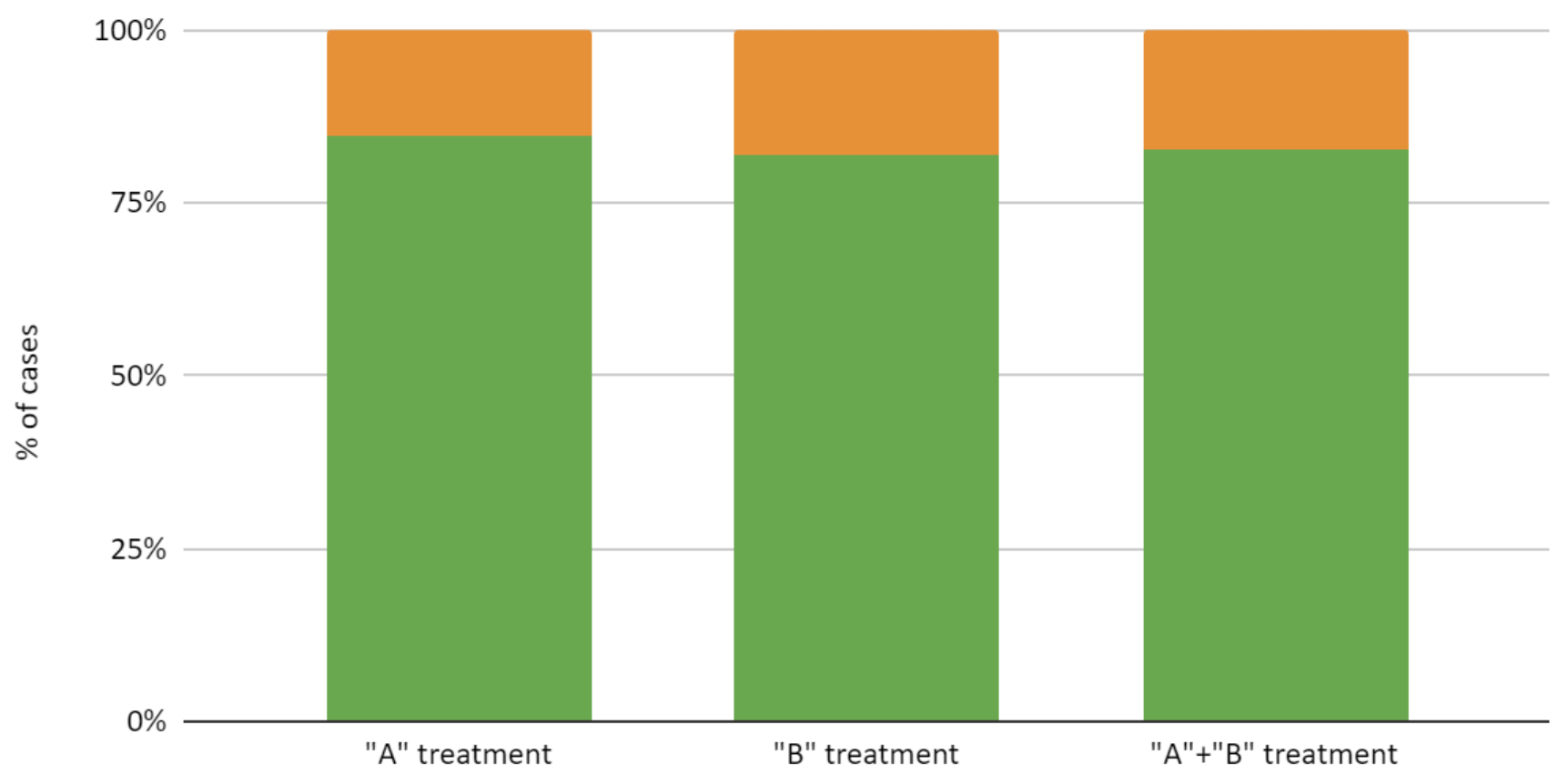

In our sample the relapse occurs in a substantially stable manner through both less invasive interventions, by debridement and remodeling, and more demanding surgery, with marginal or segmental resection. Although, as clearly evident from the data, the number of more aggressive treatments was far greater, the number of relapses remained stable and aligned in the two samples: this leads us to suppose that the risk does not lie in the type of surgery, but in the correct indication to it. 


\section{Number of relapses compared to type of surgery \\ no relapse $\square$ relapse}

80

60

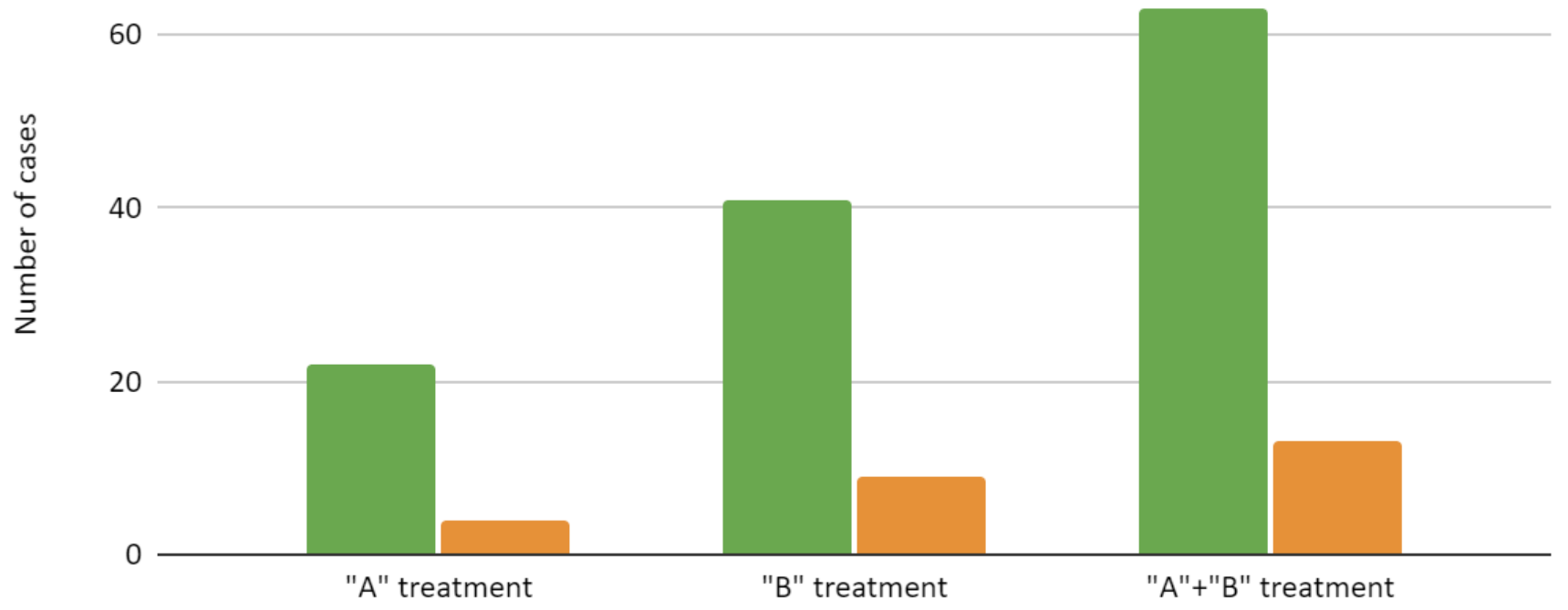

The basis of successful surgical therapy remains a watertight wound closure, but the extent of bone resection is still difficult to quantify and has been identified to be a crucial and uncertain factor. ${ }^{[18]}$ In addition, marginal resection bone bleeding might be a sign of vitality, but the reliability of this procedure remains controversial. Fluorescence-guided bone resection, based on tetracycline bone uptake, has been developed to perform selective resection providing a conservative while effective procedure . ${ }^{[19][20]}$ Despite this, data reporting long-term efficacy are still lacking.

Finally we can hypothesize, given the relative absence of data in a non-statistically significant way, a relapse trend different both from international literature, both in relation to the type of surgery performed. The evidence argues that most relapses occur in the postoperative six months. ${ }^{[21]}$ This could be made false by a data collection bias: in fact the six-month time limit of the follow up could favor a prevalence of early relapses, not reporting late ones. Even with an overestimation of recent recurrence, due to the part of the sample with follow-up still in progress, we can argue that the median and the average of relapses episodes are temporally localized in the second semester of follow-up. This fully agrees with what claimed by the sicmf sipmo statement, or the need to extend the outpatient control period at least till one year after surgery. Furthermore, we can identify a different recurrence incidence with respect to the treatment performed: type " $A$ " surgery is in fact characterized by an early peak (within three months) and a weak late recrudescence. Type "B" surgery does not present immediate complications, but rather a consistent peak after 6-12 months. This can be explained by incomplete debridement in less invasive interventions, with persistence in loco of infectious foci; in extensive resections, this may be due to the evolution of the pathology, that persists affecting the resection margins, rather than to the suffering of soft tissues, which instead clashes with a late relapse. 


\section{Number of cases compared to Months till relapse}

- "a" surgery cases = "b" surgery cases

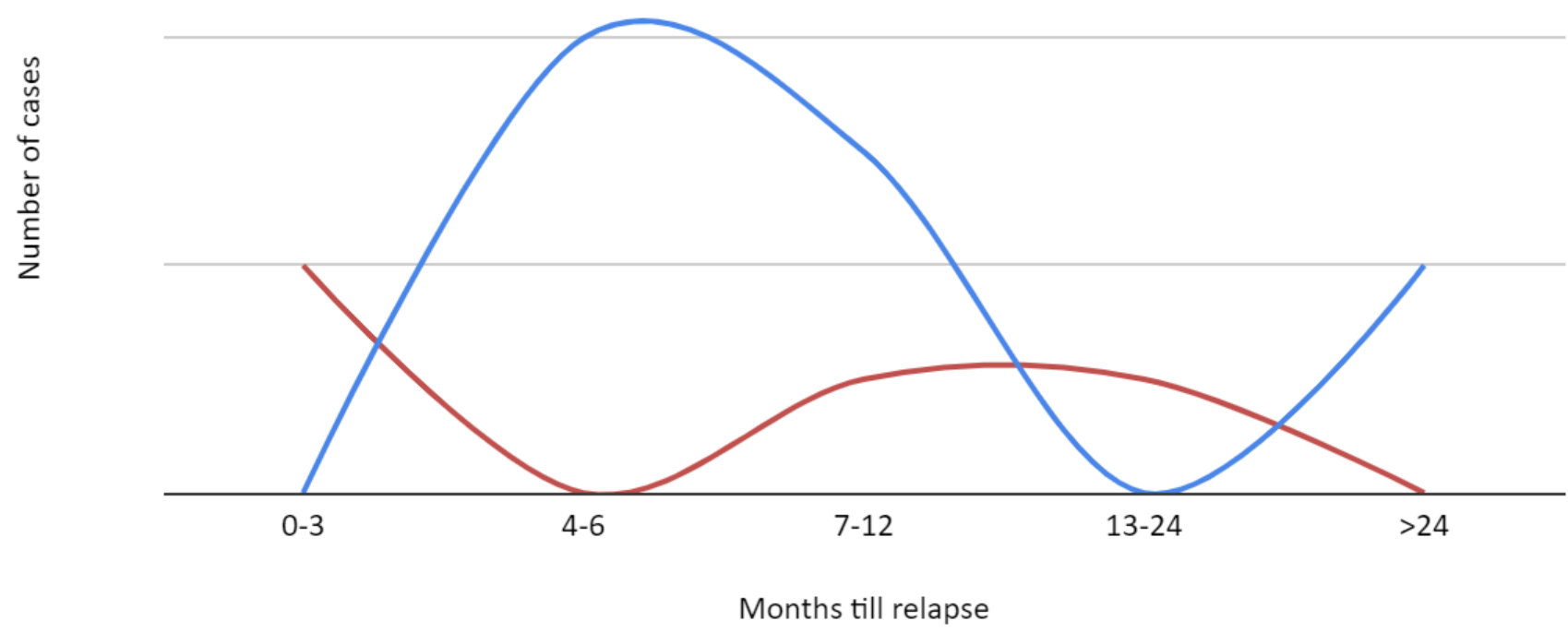

Concluding, in relation to the heterogeneity of the data, the complexity of the pathology and its particular multifactoriality, it is likely that the prognostic data, and therefore the relapse is due to the patient's specific general conditions and to the presence of any comorbidity; this has to be verified in a further study. 
ONJ examined

ONJ surgcially treated

ONJ from Oncological Pathologies 92

Relapses

13

Total surgeries performed

105

\section{Relapses}

Staging

Stage

35

Stage III

Type of surgery

"A" treatment
"B" treatment

Staging / Type of surgery

Stage I / "A" treatment
Stage II / "A" treatment
Stage III / "A" treatment
Stage I / "B" treatment
Stage II / "B" treatment
Stage III / "B" treatment

\section{Oncological pathology}

\begin{tabular}{|l|l|}
\hline Breast Cancer & 40 \\
\hline Multiple Myeloma & 36 \\
\hline Prostate Cancer & 9 \\
\hline Kidney Cancer & 4 \\
\hline Pulmunary Cancer & 1 \\
\hline Parotid Cancer & 1 \\
\hline Non-Hodgking Lymphoma & 1 \\
\hline
\end{tabular}

\section{Medication taken}

\begin{tabular}{|l|l|}
\hline Zolendronate & 71 \\
\hline Denosumab & 6 \\
\hline Zolendronate + Denosumab & 6 \\
\hline Pamindronate & 5 \\
\hline Alendronate & 4 \\
\hline
\end{tabular}

Stage I/ "A" treatment 2

Stage II / "A" treatment 2

Stage III / "A" treatment /

Stage I/ "B" treatment 2

Stage II / "B" treatment 2

Stage III / "B" treatment 5 


\section{References}

1. a, b, c G Campisi, A. Bedogni, V. Fusco.. (2020). Raccomandazioni clinico-terapeutiche sull'osteonecrosi delle ossa mascellari associata a bisfosfonati e sua prevenzione, versione 2.0. Palermo Press University.

2. `Salvatore L. Ruggiero, Thomas B. Dodson, John Fantasia, Reginald Goodday, et al. (2014). American Association of Oral and Maxillofacial Surgeons Position Paper on Medication-Related Osteonecrosis of the Jaw-2014 Update. Journal of Oral and Maxillofacial Surgery, vol. 72 (10), 1938-1956. doi:10.1016/j.joms.2014.04.031.

3. ^Waldemar Reich, Udo Bilkenroth, Johannes Schubert, Claudia Wickenhauser, et al. (2015). Surgical treatment of bisphosphonate-associated osteonecrosis: Prognostic score and long-term results. Journal of Cranio-Maxillofacial Surgery, vol. 43 (9), 1809-1822. doi:10.1016/j.jcms.2015.07.035.

4. ^Luca Ramaglia, Agostino Guida, Vincenzo lorio-Siciliano, Alessandro Cuozzo, et al. (2018). Stage-specific therapeutic strategies of medication-related osteonecrosis of the jaws: a systematic review and meta-analysis of the drug suspension protocol. Clin Oral Invest, vol. 22 (2), 597-615. doi:10.1007/s00784-017-2325-6.

5. ^Govaerts D, Piccart F, Ockerman A, Coropciuc R, Politis C, Jacobs, R. Bone. (2020). Adjuvant therapies for MRONJ: A systematic review.. Bone, vol. 2020 Dec;141:115676.

6. ^Nisi M, La Ferla F, Karapetsa D. (2016). Conservative surgical management of patients with bisphosphonate-related osteonecrosis of the jaw: a series of 120 patients.. Br J Oral Maxillofac Surg, vol. (2016) 54(8):930-935. .

7. 'Natalie H Beth-Tasdogan, Benjamin Mayer, Heba Hussein, Oliver Zolk. (2017). Interventions for managing medication-related osteonecrosis of the jaw. doi:10.1002/14651858.cd012432.pub2.

8. 'Brent O'Carrigan, Matthew HF Wong, Melina L Willson, Martin R Stockler, et al. (2017). Bisphosphonates and other bone agents for breast cancer. doi:10.1002/14651858.cd003474.pub4.

9. ^Coleman RE. (2006). Clinical features of metastatic bone disease and risk of skeletal morbidity.. Clin Cancer Res, vol. 2006;12:6243s-9s.

10. `A Limones, L-M Sáez-Alcaide, S-A Díaz-Parreño, A Helm, M-M Bornstein, P Molinero-Mourelle.. (2020). Medicationrelated osteonecrosis of the jaws (MRONJ) in cancer patients treated with denosumab VS. zoledronic acid: $A$ systematic review and meta-analysis.. Med Oral Patol Oral Cir Bucal., vol. 2020 May 1;25(3):e326-e336 .

11. ^F. Saad, J.E. Brown, C. Van Poznak, T. Ibrahim, et al. (2012). Incidence, risk factors, and outcomes of osteonecrosis of the jaw: integrated analysis from three blinded active-controlled phase III trials in cancer patients with bone metastases. Annals of Oncology, vol. 23 (5), 1341-1347. doi:10.1093/annonc/mdr435.

12. `Juliana Lemound MD, DMD, Thomas Muecke MD, DMD, Alexander-Nicolai Zeller MD, Jürgen Lichtenstein MD, DMD, André Eckardt MD, DMD e Nils-Claudius Gellrich MD, DMD. (2018). Nasolabial Flap Improves Healing in MedicationRelated Osteonecrosis of the Jaw.. Journal of Oral and Maxillofacial Surgery,, vol. 2018:76;4: 877-885, .

13. `W Reich, U Bilkenroth, J Schubert, C Wickenhauser, A W Eckert. (2015). Surgical treatment of biphosphonateassociated osteonecrosis: Prognostic score and long term results. .. J. of Cranio-Maxillo-Facial Surgery, vol. 43(2015):1809-1822.

14. ^Rodrigo Nascimento Lopes, Gustavo Davi Rabelo, Andre Caroli Rocha, Paulo Andre Gonçalves Carvalho, et al. (2015). Surgical Therapy for Bisphosphonate-Related Osteonecrosis of the Jaw: Six-Year Experience of a Single 
Institution. Journal of Oral and Maxillofacial Surgery, vol. 73 (7), 1288-1295. doi:10.1016/j.joms.2015.01.008.

15. 'S Salvatore L Ruggiero, Nina Kohn. (2015). Disease Stage and Mode of Therapy Are Important Determinants of Treatment Outcomes for Medication-Related Osteonecrosis of the Jaw .. J Oral Maxillofac Surg., vol. 2015 Dec;73(12 Suppl):S94-S100 .

16. ^Mong-Hun Kang, Dong-Keon Lee, Chang-Woo Kim, In-Seok Song, et al. (2018). Clinical characteristics and recurrence-related factors of medication-related osteonecrosis of the jaw. J Korean Assoc Oral Maxillofac Surg, vol. 44 (5), 225. doi:10.5125/jkaoms.2018.44.5.225.

17. 'AM Hinson, ER Siegel, BC Stack Jr. (2015). Temporal correlation between bisphosphonate termination and symptom resolution in osteonecrosis of the jaw: a pooled case report analysis.. J Oral Maxillofac Surg, vol. 2015 Jan;73(1):53-62.

18. `Waldemar Reich, Udo Bilkenroth, Johannes Schubert, Claudia Wickenhauser, et al. (2015). Surgical treatment of bisphosphonate-associated osteonecrosis: Prognostic score and long-term results. Journal of Cranio-Maxillofacial Surgery, vol. 43 (9), 1809-1822. doi:10.1016/j.jcms.2015.07.035.

19. 'Christoph Pautke, Florian Bauer, Oliver Bissinger, Thomas Tischer, et al. (2010). Tetracycline Bone Fluorescence: A Valuable Marker for Osteonecrosis Characterization and Therapy. Journal of Oral and Maxillofacial Surgery, vol. 68 (1), 125-129. doi:10.1016/j.joms.2009.05.442.

20. 'Kenneth E. Fleisher, Steven Doty, Shailesh Kottal, Joan Phelan, et al. (2008). Tetracycline-Guided Debridement and Cone Beam Computed Tomography for the Treatment of Bisphosphonate-Related Osteonecrosis of the Jaw: A Technical Note. Journal of Oral and Maxillofacial Surgery, vol. 66 (12), 2646-2653. doi:10.1016/j.joms.2008.07.021.

21. 'Natalie H Beth-Tasdogan, Benjamin Mayer, Heba Hussein, Oliver Zolk. (2017). Interventions for managing medication-related osteonecrosis of the jaw. doi:10.1002/14651858.cd012432.pub2. 